HEFAT2014

$10^{\text {th }}$ International Conference on Heat Transfer, Fluid Mechanics and Thermodynamics

14 - 16 July 2014

Orlando, Florida

\title{
NUMERICAL STUDY AND EXPERIMENTAL VALIDATION OF THE WATER FILMS AND THE DETACHMENT OF DROPS ON DRIFT ELIMINATORS
}

\author{
J. López ${ }^{1}$, M.D. Blanco ${ }^{1}$, A. S. Káiser ${ }^{1}$, B. Zamora ${ }^{1 *}$, A.J. Consuegro ${ }^{1}$, M. Lucas $^{2}$, J. Ruiz ${ }^{2}$ A.Viedma ${ }^{1}$ \\ *Author for correspondence \\ ${ }^{1}$ Dpto. de Ingeniería Térmica y de Fluidos. Universidad Politécnica de Cartagena. \\ ${ }^{2}$ Dpto. de Ingeniería Mecánica y Energía. Universidad Miguel Hernández \\ E-mail: blas.zamora@upct.es
}

\begin{abstract}
Water drift emitted from cooling towers is objectionable for several reasons, mainly due to human health hazards. Generation and control of drift depends mostly on the drift eliminator, a device installed in mechanical cooling towers to prevent the escape of droplets (drift). These eliminators induce a rapid alternation of direction changes, and then the droplets cannot follow the path lines of the airflow within the channels of the eliminator and impact on the plates of it, falling back to the cooling tower ground.

This paper focuses on the numerical study of a type of drift eliminator, validated by experimental tests. Three main aspects are considered: the water film formed on the plates of drift eliminators, the size of water droplets detached from this film and the condition of the detachment of these droplets. Good agreement is obtained between numerical and experimental results. The study shows that the behavior of water droplets is very influenced by the air velocity inside the cooling tower.
\end{abstract}

\section{INTRODUCTION}

Mechanical draft cooling tower are systems for heat dissipation in air conditioning installations. Its operating principle is based on the evaporation of a small part of the circulating water. The water enters the cooling tower by spray, or by free fall, with a crossflow airstream. Surface evaporation of a small part of the water induced by contact with air results in the cooling of the rest of the water falling on the raft [1].

The efficiency of cooling towers is higher than any other alternative cooling system. However, the use of these devices is limited because they can released into the environment drops of water (a phenomenon called drift), which may contain harmful chemicals and microorganisms. Drift eliminators are installed inside the tower in order to reduce the released of water drops into the environment trough the impact of these drops on drift eliminator's plates [2].

Drift eliminators are compounded by several plates placed inside the cooling tower forming angles, so that water droplets can impact them and do not follow the airstream to outside. The changes of direction formed by the plates cause the pressure loss that brings the reduction of the heat exchange with the water, or the increased of air fan's power. In any case, it results in a reduction of the global cooling system efficiency. The rate of drift loss is a function of the tower configuration, the eliminator design, the airflow rate through the tower and the water loading [2].

Several types of drift eliminators are studied in the literature to evaluate their efficiency. James et al. [3] studied numerically the behavior of two plates forming a canal with six bends. In this case, they studied a mist eliminator; a device used in the process industry to separate liquid from a gas stream, and not to heat transfer, but mist and drift eliminators operate on the same basis: liquid droplets impact on the plates, accumulate and form thin films. Zamora and Kaiser [2] evaluated the efficiency of four types of wave-plate drift eliminators with different plate forms; they also calculated numerically the droplet collection efficiency for a range of airstream velocities and droplet diameters, proposing a global correlation for this collection efficiency.

The main objective of this work is to develop a numerical procedure to reproduce the real conditions established in the crossflow between air and water in a drift eliminator placed in the interior of a cooling tower. We evaluate computationally the behavior of a type of drift eliminator in a 2D domain, by a commercial numerical code (ANSYS), comparing the obtained results with previous experimental data taken from the experimental prototype developed to that purpose. Some aspects as the water film on the plates of the eliminator, as well as the size of droplets emitted by this device are also studied.

\section{EXPERIMENTAL APPARATUS AND PROCEDURE}

\section{Installation objectives and operation}

To study the crossflow of air and water in a drift eliminator, an experimental prototype of mechanical draft cooling tower is developed. This prototype consists on a 
prismatic structure closed by 4 lateral faces, as seen on Figure 1.

Drift eliminator is placed on a test section of the experimental device. This section is a removable part of the tower in order to manage with it easily. The two steel plates forming the drift eliminator are attached to this section in a way that allows us to modify some parameters such as the distance between the plates and its slope (Figure 2).

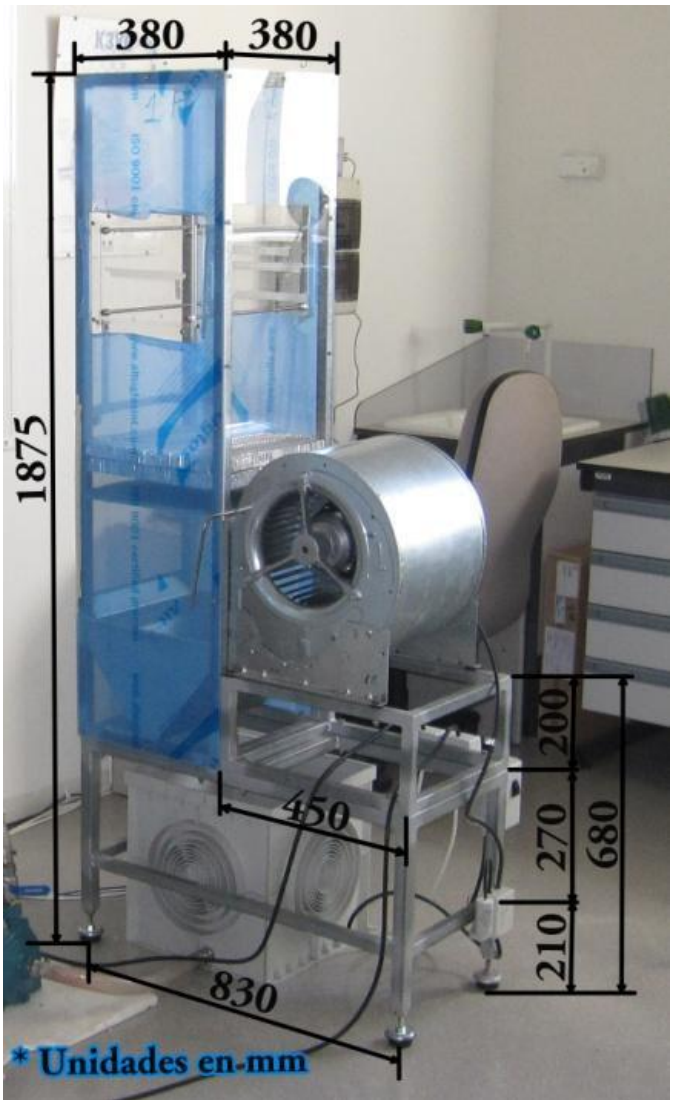

Figure 1. Illustration of the studied cooling tower installation.

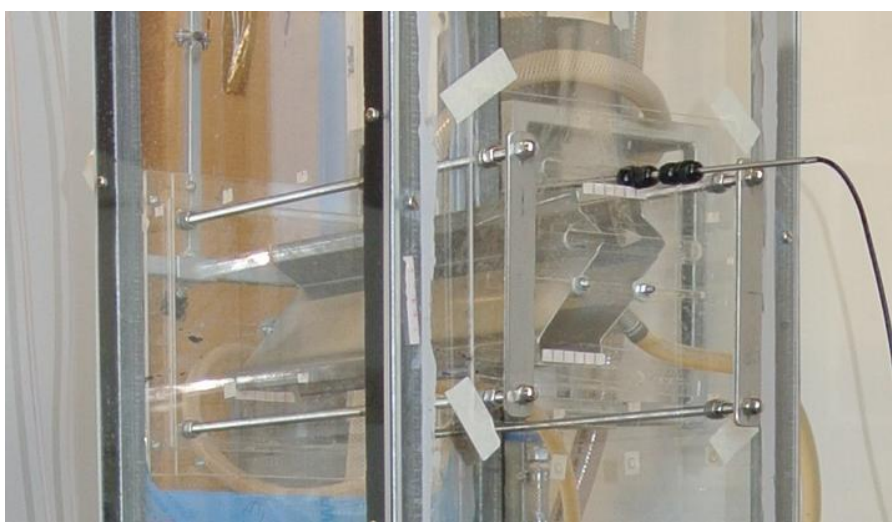

Figure 2. Image of the test section including the plates of the drift eliminator.

Water supply system is designed to maintain a constant value for water flow in the water inlet, placed on one of the plates of the drift eliminator. This system consists of two centrifugal pumps and a set of tanks: a main tank where water is stored, a double tank that supply the water to the injection system, and one tank where water is collected at the bottom of the tower.

Figure 3 shows the operating scheme of the hydraulic system. From the main tank (tank 1), water is led to tank 2.2 through pump 1, and it is supplied to the injection system; the rest of water passes first to tank 2.1 and finally reaches tank 1 . Tested water is collected at the bottom of the tower in tank 3, and it is led to tank 1 through pump 2.

In addition to this, the air supply system consists of one centrifugal fan $(0.55 \mathrm{~kW})$ and a regulator.

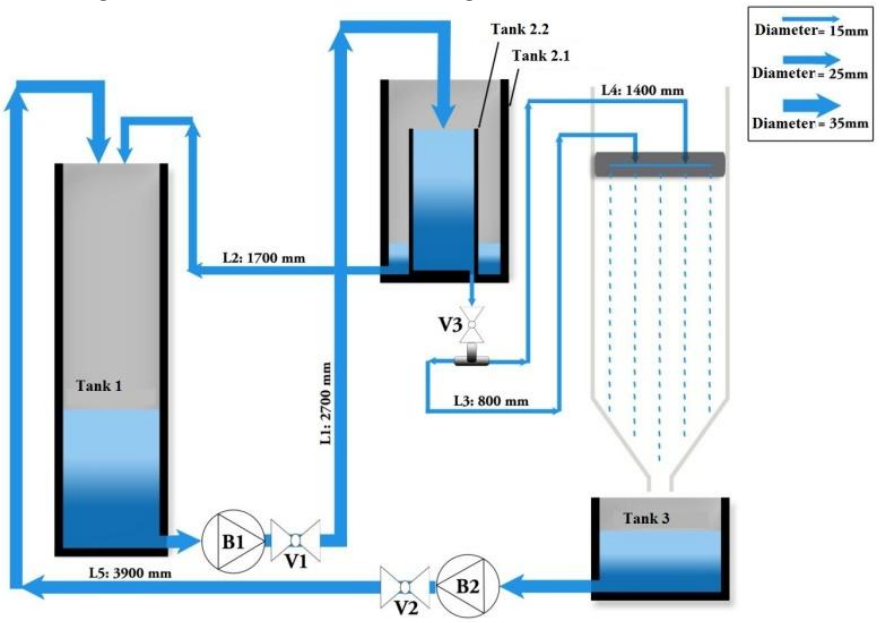

Figure 3. Operating scheme of the cooling tower.

\section{Data acquisition system and uncertainty calculation}

To determine the behavior of the cross flow between air and water in the drift eliminator, several characteristic variables of the physical process are measured. Thus, different relevant aspects like the water film formed on the plates of drift eliminators, the size of water droplets detached from this film and the condition of the detachment of these droplets, are analyzed. To measure the values for air velocity within the cooling tower, water mass flow, thickness of the water film, and the size of droplets, the equipment used is a pressure sensor for determining the height of water in tank 1 (in order to obtain the water flow), a hot wire anemometer (to measure air velocity in the test section between the drift eliminator plates), and finally a Pitot tube and a differential pressure transducer (to obtain the air velocity in a section of the tower).

All the measures are registered through a data acquisition system with a resolution of 16 bits and a maximum of 8 analogical inputs. And the images and videos needed to measure the size of droplets and the thickness of the film are taken by a high velocity camera (maximum velocity $1200 \mathrm{fps}$ ).

To calculate the uncertainty of the measurement process, the reference guide "A Guide to Expression of Uncertainty in Measurement" (GUM), ISO/1995 was followed. The class A uncertainty is obtained by statistical methods from experimental values, whereas the class B uncertainty takes into account errors of each sensor. Total uncertainty is a combination of these two types. Uncertainty of the used equipment is shown in Table 1. 


\begin{tabular}{|c|c|c|}
\hline Equipment & Measure & Uncertainty \\
\hline Pressure sensor \\
Siemens Sitrans \\
PMPS & Height of water in tank & $1.73 \mathrm{~mm}$ \\
\hline Wot wire anemometer & Water flow & $0.0561 / \mathrm{min}$ \\
TSI AVT & Air velocity* & $0.095 \mathrm{~m} / \mathrm{s}$ \\
& & $0.138 \mathrm{~m} / \mathrm{s}$ \\
Pitot tube SR 305 & Air velocity & $0.196 \mathrm{~m} / \mathrm{s}$ \\
\hline
\end{tabular}

Table 1. Uncertainty of used equipments. *Depends on the chosen scale.

\section{Experimental tests plan}

First of all, tests are carried out without water in order to measure the air velocity field in the test section. Measures are taken with the hot wire anemometer at three different heights inside the channel formed by the two plates (h1, h2 and h3) and at five different points (Figure 3 ) distanced by $10 \mathrm{~mm}$ covering the whole gap between the plates $(60 \mathrm{~mm})$. Therefore, it represents 15 control points of measurements.

Different air velocities are studied in the tests: V0 (with the fan switched off), V1, V2, V3 and V4 (2, 4, 6 and $8 \mathrm{~m} / \mathrm{s}$ respectively at the air inlet section). Four values of the water mass flow are considered to be experimentally tested: Q1, Q2, Q3 and Q4 (0.14, 0.28, 0.67 and 2.91 1/min respectively). Therefore, it represents 16 different experimental cases analyzed.

For each of these values of water mass flow, two limits values for the air velocity should be determined: the first one is called VL1. This air velocity is the one that causes the detachment of droplets at the lower zone of the drift eliminator. The second is VL2, the air velocity that causes the detachment of droplets at the upper zone of the drift eliminator. Both zones are marked in Figure 3 with a red circle.

\section{NUMERICAL MODELING}

\section{Computational domain}

The 2D cooling tower modeling was carried out with the ANSYS DesignModeler software. The computational domain is a rectangle of 880 × $330 \mathrm{~mm}$ (Figure 3). Structured, nonuniform meshes are employed to obtain the numerical results. Taking into account that the water film thickness is lower than $1 \mathrm{~mm}$ in many cases and the flow front advance changes with the time (it is a transient problem), prohibitive computational resources are needed to tackle this problem. Therefore, the ANSYS Fluent meshing adaption option is employed to refine the mesh in zones where water appears in a volume fraction over 0.3 . Figure 4 shows the mesh before including water. In this way, the selected mesh corresponds to a relationship of compromise between the number of elements and the accuracy in the simulation, and it is composed by around 200,000 cells.

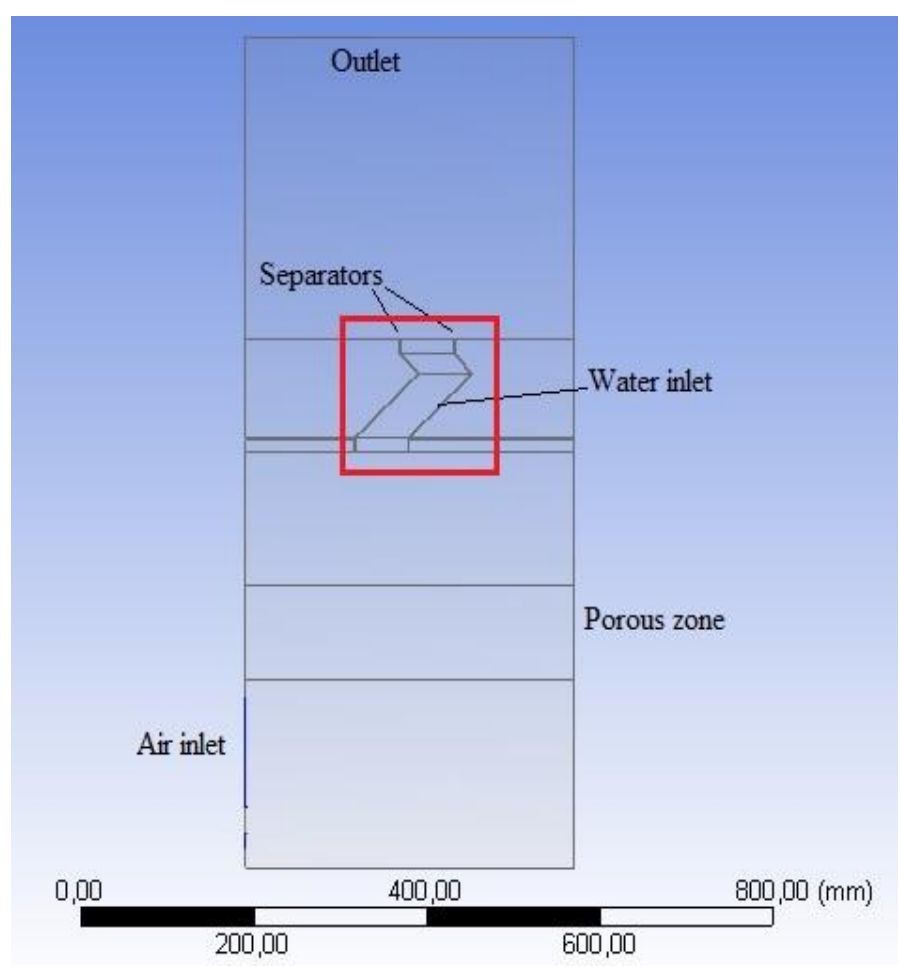

a)

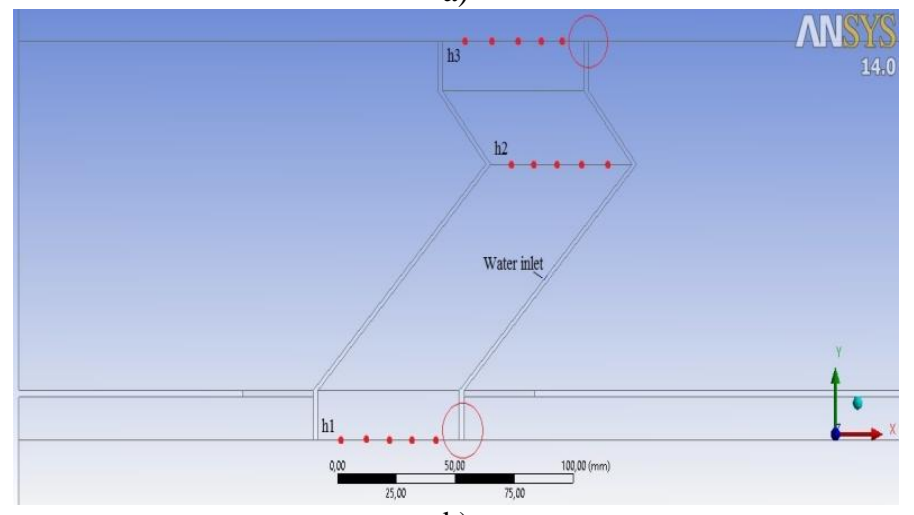

b)

Figure 3. (a) Schematic representation of the cooling tower. (b) Schematic representation of the channel zone; points indicate the points where experimental measures were taken at h1, h2 and h3; circles mark the edges where detachment of droplets happens.

\section{Boundary conditions}

Regarding on boundary conditions, the pressure outlet condition is set on the upper base of the cooling tower with a zero value for gauge pressure. For air entrance, velocity inlet condition is used, with velocity values chosen in order to adjust airstream velocities in the channel zone comparing to experimental results. Finally, to water injection, also a velocity inlet condition is used; the water injection surface in the installation is a $2 \times 275 \mathrm{~mm}$ rectangle, so we obtain the velocity value by dividing the experimental value of water flow by the area of the surface.

Another aspect considered is the porous zone installed inside the cooling tower. This zone is defined as a $100 \mathrm{~mm}$ section of the tower, placed right over the air entrance. This zone allow us obtaining the right airstream velocities in the 
channel, apart from that, the air velocity field become uniform while passing through the porous zone. In $2 \mathrm{D}$ simulations, the porous zone is defined as a region with a 0.8 value for porosity.
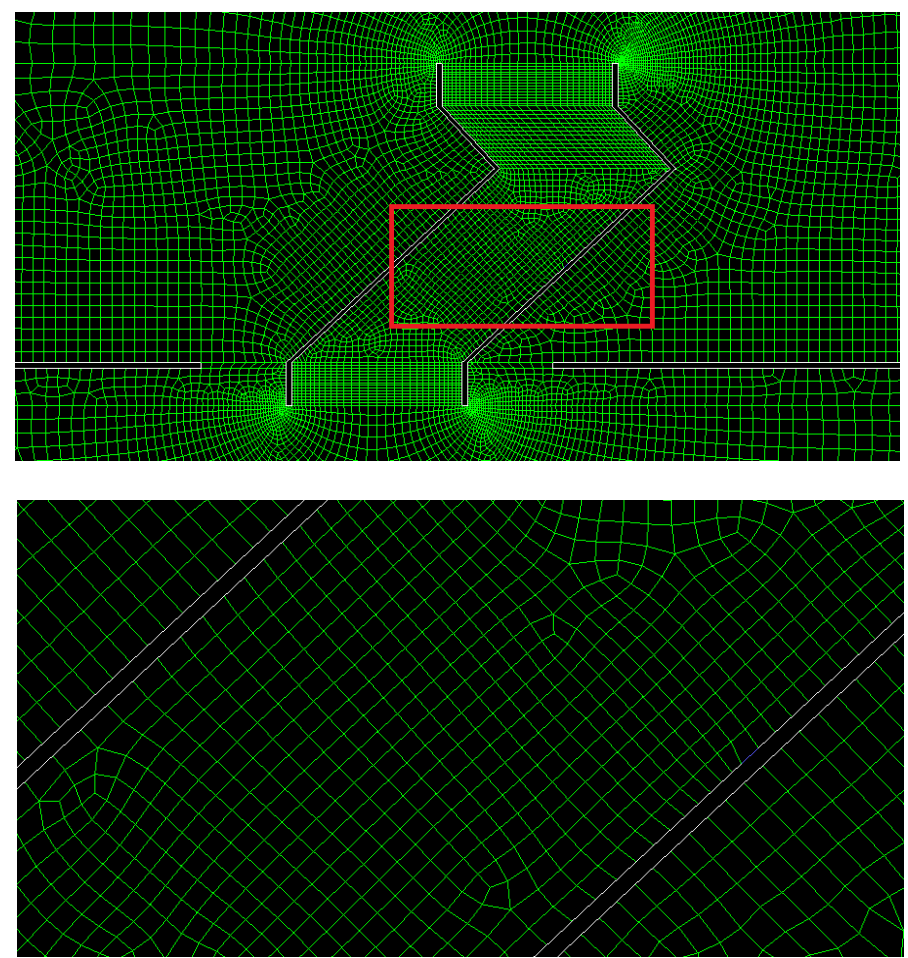

Figure 4. (a) Mesh used for the channel zone. (b) Detail of element size in the channel zone, note that the thickness of plates is $2 \mathrm{~mm}$.

\section{Numerical approach}

Steady state simulations without water are done at every air velocity. The standard $k-\varepsilon$ turbulence model is employed in order to simulate the turbulence of the airstream; in cases without water, we obtain a proper approximation to experimental data.

Regarding the numerical convergence, the relative change of any dependent variable in each iteration was lower than $10^{-5}$, besides the normalized residuals for mass, momentum, and turbulent variables for the full flow field had to be below $10^{-5}$ also.

To simulate multi-phase flow, the Volume-of-Fluid Model (VOF) was used. The VOF model can simulate two or more immiscible fluids by solving a single set of momentum equations and tracking the volume fraction of each of the fluids throughout the domain. This model can be only used with the pressure-based solver. With regard to numerical convergence, for each time step, the criterion is a value for the continuity residual of $5 \times 10^{-6}$.

Surface tension effects between air and water are also considered in the simulations. Wall adhesion is enabled and a value of $0.072 \mathrm{~N} / \mathrm{m}$ is fixed for surface tension. Moreover, the contact angle between water, air and the plate is set in $45^{\circ}$ and the roughness of the plate is considered. Both contact angle and wall roughness were adjusted so the numerical and experimental results fit as well as possible.
Volume-of-Fluid Method needs a specific solution method, such as geo-reconstruct, CICSAM, compressive, QUICK or modified HRIC. In this problem, CICSAM solution method is used because it is a high resolution differencing scheme, particularly suitable for flows with high ratios of viscosities between the phases.

Once the simulation with just air is converged in a steady state simulation, then a transient simulation is carried out; the Courant number is one of the main problems to solve in order to achieve a proper simulation; it relates the size of cells forming the interface with the time step size. The Courant number is set in 0.01 and the initial time step size is $8 \cdot 10^{-6}$ seconds, both parameters were set through a number of previous testing simulations, in order to reach the proper solution in an optimal computation time. The time step size is so small due to the size of the cells in the interface zone (less than 500 microns).

\section{RESULTS AND DISCUSSION}

A comparison of the experimental and numerical results for air velocity field, water film thickness, size of droplets and the conditions that cause the detachment of droplets is shown in the following figures and tables.

\section{Air velocity fields}

First of all, a validation for airstream velocities is done with the previous experimental data obtained in the prototype about air velocities in the channel zone, and the numerical results in the same sections of the channel. Figure 5 shows an example of the numerical results for the air velocity magnitude field; in this figure, the zones with higher velocities can be seen.

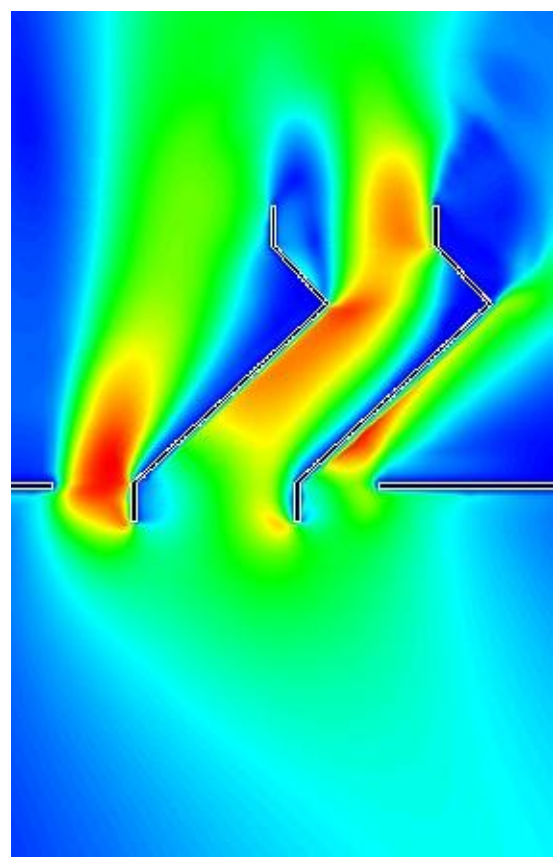

Figure 5. Representative velocity field in the test section. 
Figures 6 to 9 show that a good agreement is achieved between the numerical and the experimental results for velocity magnitude. Trends are similar in both cases and also the values for air velocity with a mean error of $20 \%$, despite of random component in turbulent flows and other uncertainties.

In these figures air velocity magnitude is shown in the channel zone where experimental measures have been taken. The velocity inlet boundary condition was obtained by testing air velocities in the inlet and comparing the result with the experimental one.

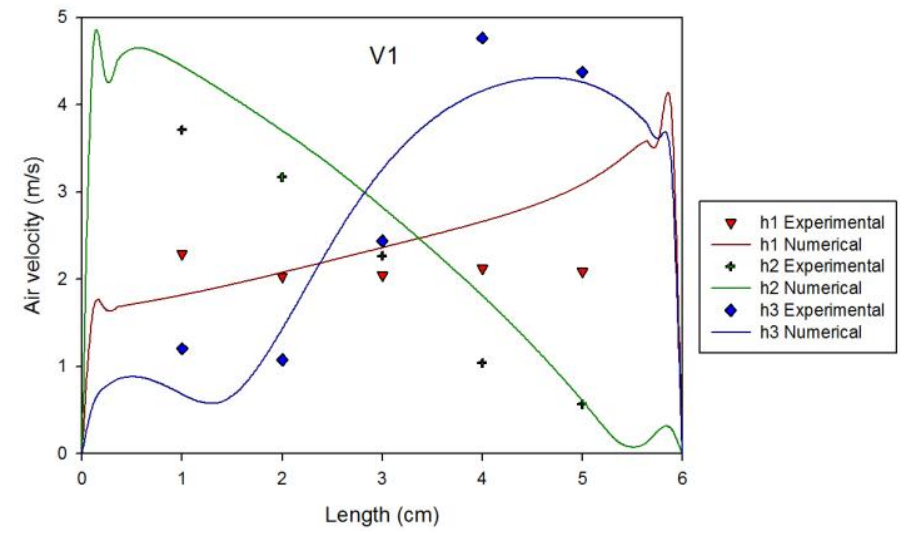

Figure 6. Air velocities for V1 at the air entrance section.

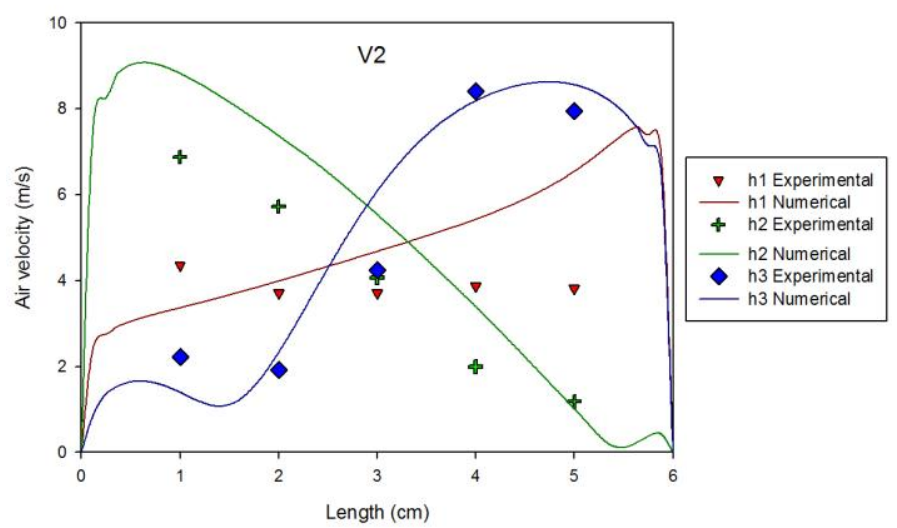

Figure 7. Air velocities for V2 at the air entrance section.

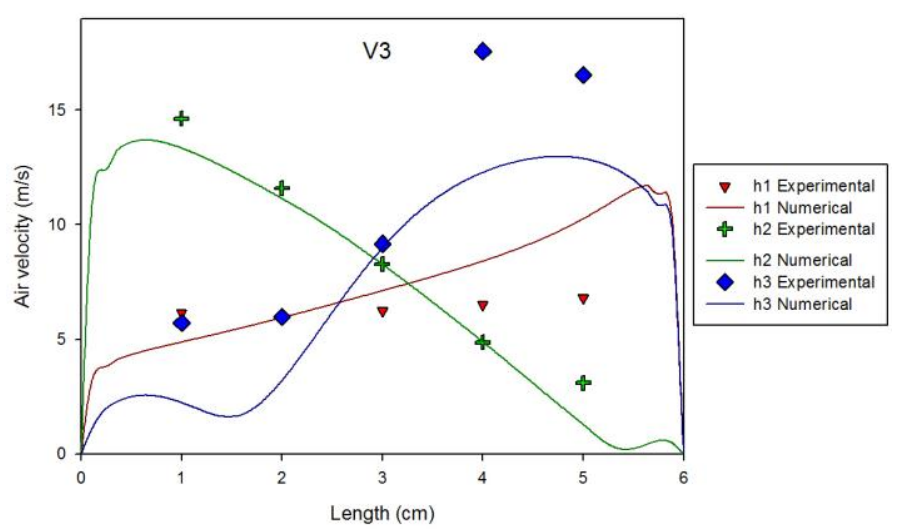

Figure 8. Air velocities for $\mathrm{V} 3$ at the air entrance section.

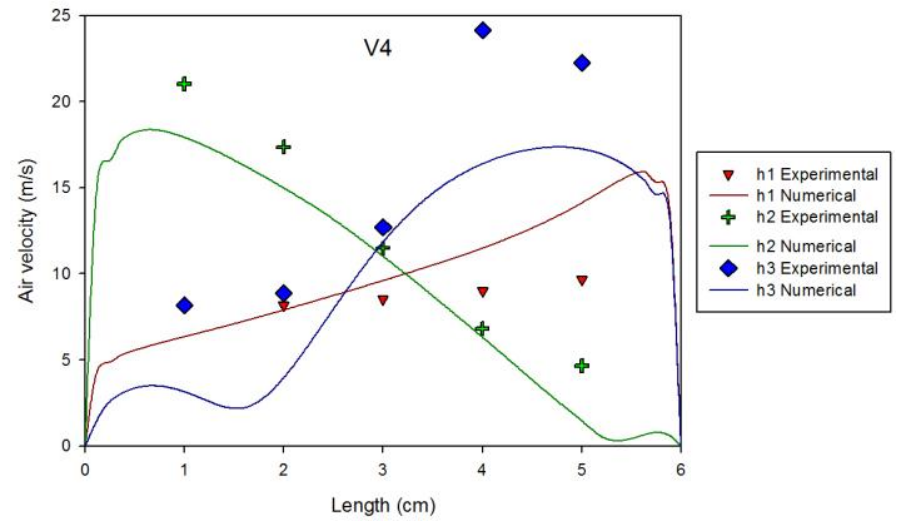

Figure 9. Air velocities for V4 at the air entrance section.

\section{Water film and detached droplets}

Once the airstream is fully developed, the secondary phase is included in the problem.

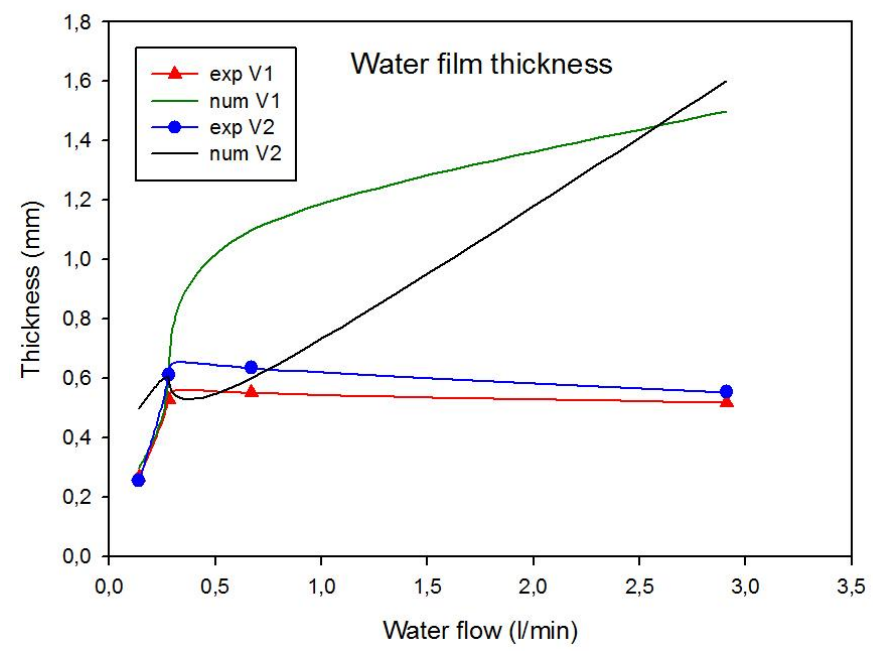

Figure 10. Water film thickness in cases for V1 and V2 and the four different values for water flow.

In Figure 10, trends for water film thickness are shown. The air velocities chosen for this plot are the lower ones (V1 and V2). We can see that values for water flows Q1, Q2 and Q3 are similar in both experimental and numerical cases. Some differences appear when testing the largest water flow.

The used images are taken from one side of the tower, as seen on Figure 11, capturing the whole thickness of the film. Regarding numerical case, the interface criterion is set to 0.5 of water volume fraction so that the quantity of water included in the film is enough to get an accurate solution [4]. 

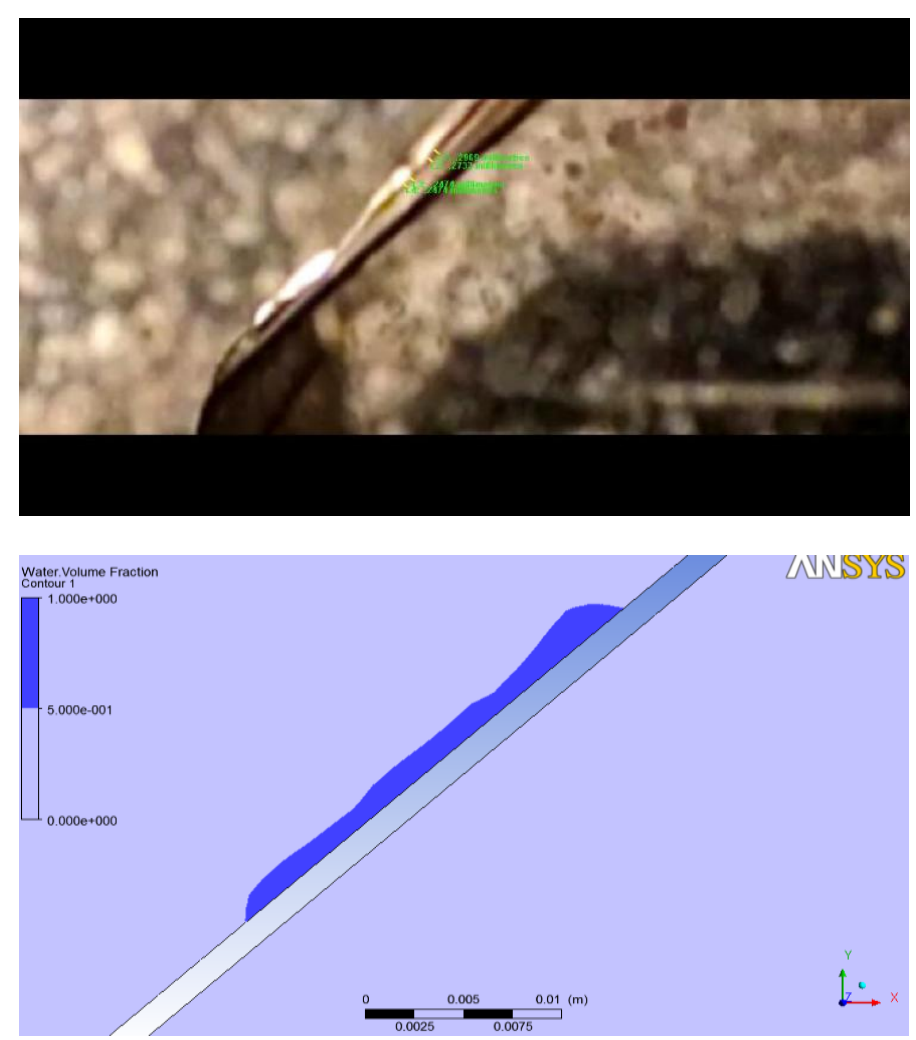

Figure 11. Images of experimental and numerical water film over the plate.

Figure 12 represents examples of images used for droplet measurement in experimental and numerical tests. Also results for experimental droplet size are shown, as well as some results for numerical droplet size in order to compare both cases.

Table 2 shows that a good agreement between experimental data and numerical solution exists; note that trends are similar and droplet sizes have a mean error of $25 \%$. In cases with high air velocities, water film breaks into smaller droplets due to the higher shear forces of air over the water surface. Otherwise, droplets are higher when water flow increases, as well as the water film thickness. This thickness is also higher when air velocity increases; the explanation for that is based on the force that airstream performs on the water film. That force slows down the water and makes its thickness increase.

\begin{tabular}{|c|c|c|c|c|c|}
\cline { 3 - 6 } \multicolumn{2}{c|}{} & Q1 & Q2 & Q3 & Q4 \\
\hline \multirow{2}{*}{ V1 } & Experimental & 6,39 & 6,75 & 7,16 & $*$ \\
& Numerical & 5 & 6 & 11 & 16 \\
\hline \multirow{2}{*}{ V2 } & Experimental & 8,24 & 8,93 & 9,36 & 9,56 \\
& Numerical & $* *$ & $* *$ & 10 & 16 \\
\hline
\end{tabular}

Table 2. Droplet diameter in $\mathrm{mm}$ for droplets falling from the separator plate. $(*)$ indicates difficulties to measure a single drop. (**) indicates huge computational cost.

\begin{tabular}{|c|c|c|c|c|c|}
\cline { 3 - 6 } \multicolumn{2}{c|}{} & Q1 & Q2 & Q3 & Q4 \\
\hline V3 & Experimental & 1,96 & 4,1 & 4,29 & 4,29 \\
\hline V4 & Experimental & $*$ & 3,31 & 3,01 & 2,94 \\
\hline
\end{tabular}

Table 3. Droplet diameter in $\mathrm{mm}$ for droplets leaving the separator in the upper zone. $(*)$ indicates difficulties to measure a single drop.
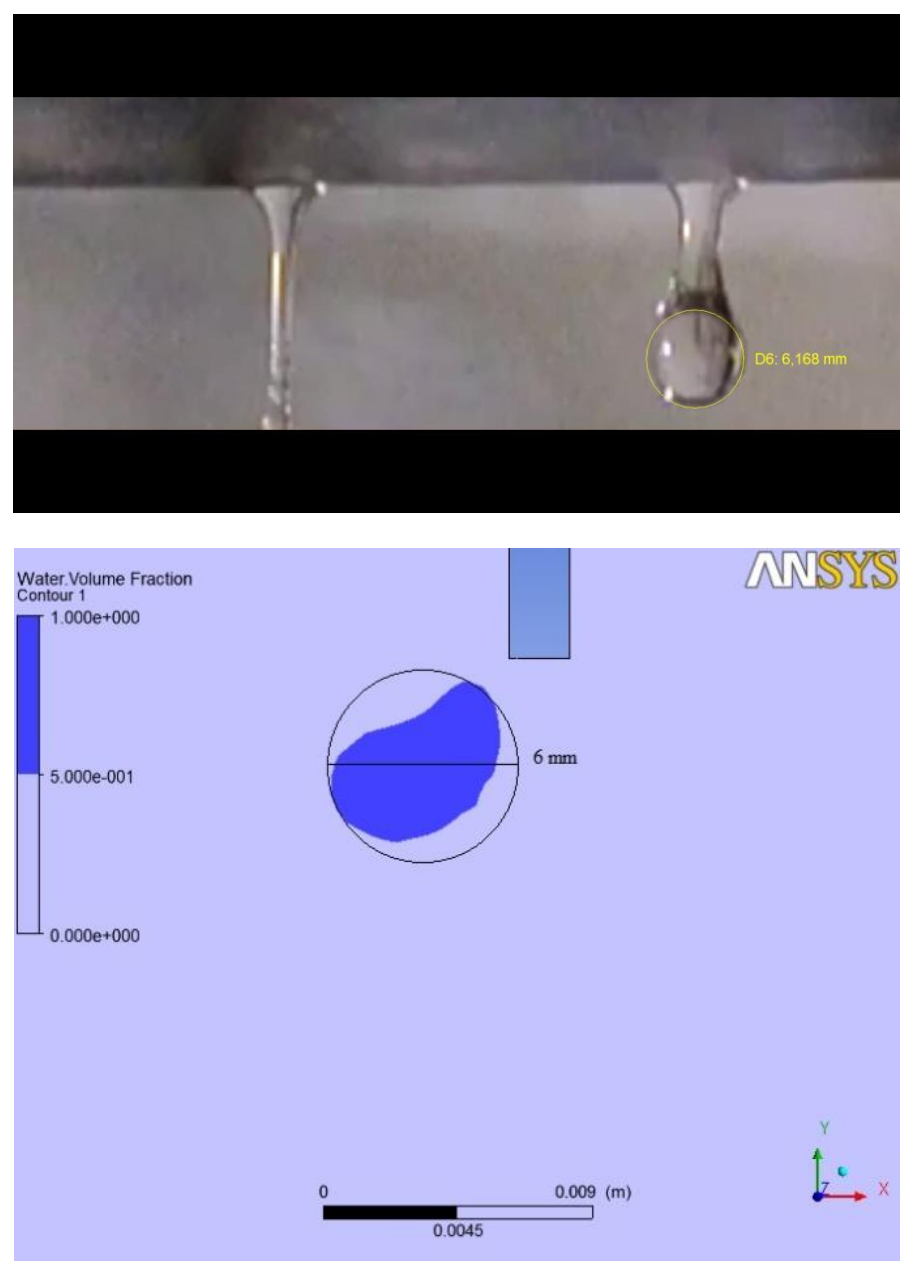

Figure 12. Images of experimental and numerical droplets leaving the separator plate. The experimental case is the V1Q1 and the numerical one is the V1Q2.

\section{Reentrainment of water film}

Results for air velocities, water film thickness or droplet size are useful in order to validate the numerical model; However, what is more relevant is to determine the maximum air velocity that can be set in the cooling tower, so that water do not release into the environment. Experimentally, tests for all water flows have been done. There are two different possibilities: when the water film reaches the upper edge (VL2) of the separator plate or when the water reaches the lower one (VL1). In both cases water drops can detach from the film and fall down, or detach and release into the environment. A limit 
air velocity exists for any case as shown in Table 4 . When air velocity is higher than VL2, then water releases into the environment.

\begin{tabular}{|l|l|l|}
\hline & VL1 $(\mathrm{m} / \mathrm{s})$ & VL2 $(\mathrm{m} / \mathrm{s})$ \\
\hline Q1 & 4.55 & 13.71 \\
Q2 & 4.36 & 12.98 \\
Q3 & 4.34 & 11.30 \\
Q4 & 4.34 & 12.50 \\
\hline
\end{tabular}

Table 4. Limit air velocities for every water flow, where VL1 represents the cases when water reaches the lower edge of the plate; and VL2 when water reaches the upper edge.

Numerical simulations have been carried out in order to compare this point. Figure 13 represents the Q4 case; it means the higher value for water flow. Limit velocities in experiments were measured in the middle point of the line that links both plates; so in numerical simulations, we have to try some different values for air velocity at the inlet boundary condition.

To obtain the limit velocity, several simulations are carried out with the same value for the water flow and trying different air velocities at the boundary condition until the detachment of droplets happens. A final value of $8.5 \mathrm{~m} / \mathrm{s}$ is reached at the air inlet; this value causes an air velocity of $12.6 \mathrm{~m} / \mathrm{s}$ in the point where experimental measures are taken, in Table 4, the value for VL2 and Q4 is $12.5 \mathrm{~m} / \mathrm{s}(0.8 \%$ error).

Figure 13 shows how water drops detached from the film leave the plate and enter the main airstream to get out of the tower.

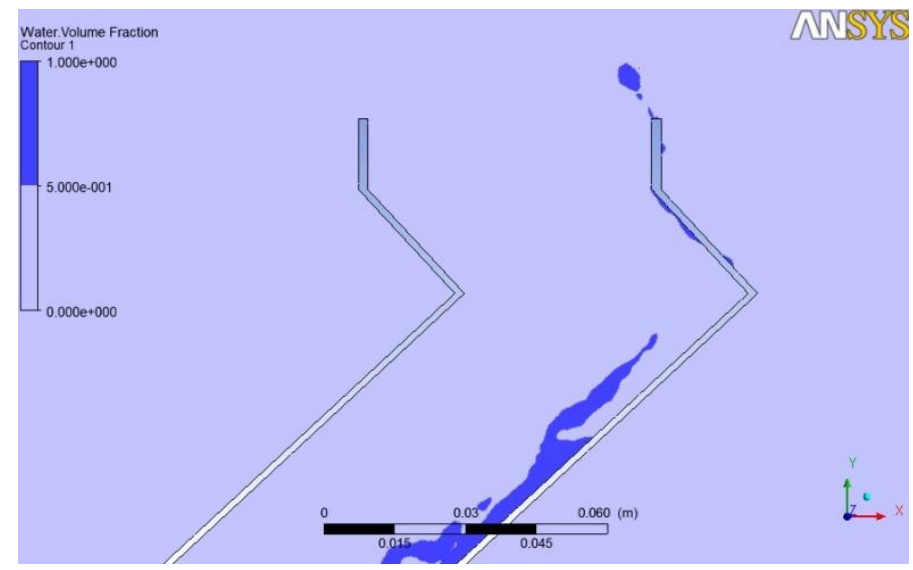

Figure 13. Water volume fraction for VL2-Q4 case $(8.5 \mathrm{~m} / \mathrm{s})$.

\section{Conclusions}

The numerical model for simulating the water films and the detachment of drops on drifts eliminators has been experimentally validated. The presented results are similar in numerical and experimental cases, and the trends in air velocity, water film thickness and droplet size are mainly the same. Accurate results have been reached for the reentrainment of water drops problem, which is the most important commercial issue considered in this paper.

Future goals will be studying 3D effects on the numerical model.

\section{ACKNOWLEDGMENTS}

This research is sponsored by the Spanish Government (Projects ENE2010-21679-C02-01 and -02), as well as by the Seneca-Agency for Science and Technology of the Region of Murcia, Spain (Project 15184/PI/10).

\section{REFERENCES}

[1] M. Lucas, J. Ruíz, P. J. Martínez, A. S. Kaiser, A. Viedma, B. Zamora. Experimental study on the performance of a mechanical cooling tower fitted with different types of water distribution systems and drift eliminators, Applied Thermal Engineering, Vol. 50, 2013, pp. 282-292.

[2] B. Zamora, A.S. Kaiser, Comparative efficiency evaluations of four types of cooling tower drift eliminator, by numerical investigation, Chemical Engineering Science, Vol. 66, 2011, pp. 1232-1245.

[3] P.W. James, B.J. Azzopardi, Y. Wang, J.P. Hughes. A model for liquid film flow and separation in a wave-plate mist eliminator, Chemical Engineering Research and Design, Vol. 83, 2005, pp. 469477.

[4] H. Lan, M. Friedrich, B. F. Armaly, J. A. Drallmeier. Simulation and measurement of 3D shear-driven thin liquid film flow in a duct, International Journal of Heat and Fluid Flow, Vol. 29, 2008, pp. 449-459.

[5] ANSYS Fluent Theory Guide, www.ansys.com. 